\title{
Vibrio harveyi protease deletion mutant as a live attenuated vaccine candidate against vibriosis and transcriptome profiling following vaccination for Epinephelus fuscoguttatus
}

\begin{abstract}
Grouper aquaculture industries have a high risk of being inflicted by bacterial diseases such as vibriosis. Various types of vaccines for vibriosis have been studied throughout the years, yet the potential of live attenuated vaccines remains unsubstantial. Correspondingly, this study attempts to develop a Vibrio harveyi protease deletion mutant into a live attenuated vaccine candidate against vibriosis for Epinephelus fuscoguttatus. Site-directed mutagenesis (SDM) and allelic exchange replacement techniques are employed to synthesize genetically attenuated V. harveyi strain MVh-vhs. The evaluation on safety levels showed that MVh-vhs strain is safe when tested on E. fuscoguttatus. A $100 \%$ survival rate with no sign of vibriosis is indicated in fish challenged with the attenuated strain. In contrast, fish challenged with the parental strain showed obvious clinical signs of vibriosis. The median lethal dosage (LD50) of fish challenged with the parental strain is found at $10^{6} \mathrm{CFU} / \mathrm{fish}$. A single dose IP administration of the attenuated strain at $107 \mathrm{CFU} /$ fish following a bacterial challenge at dose $10^{8} \mathrm{CFU} /$ fish is done 4 weeks post vaccination. The vaccinated fish show $52 \%$ relative percentage survival (RPS). The transcriptomic profiling following vaccination evoked the regulation of autophagosome pathway and the coagulation and complement cascade pathways as well as antigen processing and presentation pathways. As a conclusion, the V. harveyi attenuated strain MVh-vhs has significant potential to be applied as a live vaccine candidate against vibriosis for E. fuscoguttatus.
\end{abstract}

Keyword: Epinephelus fuscoguttatus; Fish immunity; Live attenuated vaccine; Vibrio sis; Vibrio harveyi 